PROCEEDINGS OF THE AMERICAN MATHEMATICAL SOCIETY

Volume 126, Number 3, March 1998, Pages 653-656

S $0002-9939(98) 04131-8$

\title{
NIL SUBSETS OF GRADED ALGEBRAS
}

\author{
S. MONTGOMERY AND L. W. SMALL \\ (Communicated by Ken Goodearl)
}

\begin{abstract}
We prove that if $A$ is a Noetherian $\mathbf{Z}$-graded algebra, then the Jacobson radical of $A$ is nilpotent under mild hypotheses on $A_{0}$. We also consider affine PI-algebras graded by torsion groups. Finally we prove a Nullstellensatztype theorem for enveloping algebras of Lie color algebras.
\end{abstract}

\section{INTRODUCTION}

In this paper we study various subsets of a graded algebra $A$ by using Jacobson's notion of weakly-closed subsets. When $A$ is Noetherian, a result of Jacobson-Goldie says that weakly-closed nil subsets are nilpotent; as a consequence we prove that when $A$ is $\mathbf{Z}$-graded, the Jacobson radical $J(A)$ of $A$ is nilpotent provided that $J(A) \cap A_{0}$ is nil. We also show that if $A$ is an affine PI-algebra graded by a torsion group $G$, then $A$ is a finite module over its identity component $A_{e}$ whenever $A_{e}$ is central.

We then consider some applications to the enveloping algebra of a Lie color algebra $L$. We show that when $L$ is finite-dimensional, then the endomorphism ring of an $L$-module of finite length is finite-dimensional; this is a kind of generalized Hilbert Nullstellensatz, and extends the analogous result for ordinary Lie algebras. We also give a short proof of an Engel-type result of [BG].

Throughout, $A$ denotes an algebra over the commutative ring $k$. Usually $A$ is graded by the group $G$; thus $A=\oplus_{g \in G} A_{g}$, where the $A_{g}$ are $k$-submodules of $A$ and $A_{g} A_{h} \subseteq A_{g h}$. Following [J], we say that a subset $S$ of $A$ is weakly-closed if for any pair $s, t \in S$, there exists $\gamma=\gamma(s, t) \in k$ such that $s t+\gamma(s, t) t s \in S$. Jacobson proves that when $A$ is right Artinian, the (associative) subalgebra of $A$ generated by a nil weakly-closed subset is nilpotent [J, p. 201].

\section{JACOBSON RADICALS}

In this section we show that the Jacobson radicals of certain graded rings are nilpotent. Our first result is a strengthening of Jacobson's result due to Goldie.

Proposition $1.1([\mathrm{G}])$. A nil weakly closed subset of a Noetherian algebra generates a nilpotent subalgebra.

Received by the editors June 17, 1996 and, in revised form, August 23, 1996.

1991 Mathematics Subject Classification. Primary 16N40, 16W40, 16P40; Secondary 16S30.

Both authors were supported by the NSF. 
We note that 1.1 follows by standard arguments: if $N$ is the nilpotent radical of the algebra $A$, then one may apply Jacobson's result to the ring of fractions of the semiprime Noetherian ring $A / N$.

Corollary 1.2. Let $A$ be a Noetherian algebra, graded by the group $G$, and let $B$ be a graded subalgebra of $A$ such that the homogeneous elements of $B$ are nilpotent. Then $B$ is nilpotent.

Proof. Let $S$ be the set of homogeneous elements in $B$. Then $S$ is a nil weakly closed subset of $A$, and so is nilpotent by Proposition 1.1.

But then $B$ is nilpotent.

We note an easy consequence of the corollary: if $A$ is Noetherian and gradedsemiprime, then any non-zero graded left or right ideal contains a non-nilpotent homogeneous element. This condition is used in $[\mathrm{NvO}]$ in proving a graded version of Goldie's theorem.

We also note that Corollary 1.2 is really special to the graded case; it is not sufficient to assume that every element in $B$ is a sum of nilpotent elements. For consider the ring $R$ of $n \times n$ matrices $(n>1)$ over a division ring $D$ such that every element of $D$ is a sum of additive commutators; then every element of $R$ is a sum of nilpotent elements, but $R$ is certainly not nilpotent $[\mathrm{H}]$.

We can now prove the main result of this section. We let $J(A)$ denote the Jacobson radical of $A$.

Theorem 1.3. Let $A$ be a Noetherian algebra, graded by $\mathbf{Z}$, and assume that $J(A) \cap$ $A_{0}$ is nil. Then $J(A)$ is nilpotent.

Proof. By a result of Bergman (see [P, p. 225]), $J(A)$ is a graded ideal and each $J(A)_{m}$ is nil, for $m \neq 0$. By hypothesis, also $J(A)_{0}$ is nil. Thus $B=J(A)$ satisfies the hypotheses of Corollary 1.2, and so is nilpotent.

Since $J(A) \cap A_{0} \subseteq J\left(A_{0}\right)$, the hypothesis of the theorem will be satisfied whenever $J\left(A_{0}\right)$ is nil.

If an algebra $A$ is graded by a finite group $G$, a similar result is shown in [CR, Lemma 1] with no finiteness hypotheses on $A$ : if $B$ is a graded subalgebra such that $B_{e}$ is nilpotent, then $B$ is nilpotent.

\section{AfFine Pi ALGEBRAS}

In this section we give some sufficient conditions for an algebra $A$, graded by a torsion group $G$, to be a finite module over $A_{e}$.

Theorem 2.1. Let $A$ be a $k$-affine PI algebra, graded by the torsion group $G$, such that $A_{e}$ is central. Then $A$ is a finite module over $A_{e}$ and consequently $A_{e}$ is $k$-affine. If also $k$ is Noetherian, then both $A$ and $A_{e}$ are Noetherian.

Proof. We may assume that $A$ is generated by homogeneous elements. Since $G$ is torsion, for each $g \in G$, there is some $n>0$ such that $g^{n}=e$, and thus if $a \in A_{g}$, we have $a^{n} \in A_{e}$.

Now define $B_{0}:=A_{e}$. Then $A=B_{0}\left[a_{1}, \ldots, a_{t}\right]$, where the $a_{i}$ are homogeneous; we may assume that $a_{1}=1$. We consider $A$ as a filtered algebra, as follows: define $B_{1}:=a_{1} B_{0}+\cdots+a_{t} B_{0}$ and $B_{m}:=B_{1}{ }^{m}$, for all $m>0$ (we assume $B_{1}{ }^{0}=B_{0}$ ). Then $A=\bigcup_{m \geq 0} B_{m}$. We may then form the associated graded algebra

$$
G r(A)=\oplus_{m \geq 0} B_{m} / B_{m-1},
$$


where $B_{-1}:=0$. In $G r(A)$, let $S$ be the semigroup generated by those $\bar{a}_{i}=a_{i}+B_{0} \in$ $\bar{B}_{1}=B_{1} / B_{0}$ such that $a_{i} \notin B_{0}=A_{e}$. Then $S$ is weakly closed (as it is closed under ordinary products) and $S$ is nil. For, consider any monomial $\bar{a}_{i_{1}} \cdots \bar{a}_{i_{k}}$ in $S$. Now in $A, b=a_{i_{1}} \cdots a_{i_{k}}$ is homogeneous, and thus $b^{n} \in A_{e}$ for some $n$, as noted above. Thus in $\operatorname{Gr}(A),\left(\bar{a}_{i_{1}} \cdots \bar{a}_{i_{k}}\right)^{n}=\overline{0}$.

Now $\operatorname{Gr}(A)$ is also a PI ring, and so it follows that $S$ is nilpotent, by standard arguments ( if not, there exists a prime ideal $P$ so that $S^{k} \nsubseteq \subseteq$ for all $k$; then pass to $\operatorname{Gr}(A) / P)$.

But then $\bar{B}_{m}=0$ for all $m \geq M$, for some $M$. Thus $\operatorname{Gr}(A)$ is a finite module over $B_{0}$, and so $A$ itself is a finite module over $B_{0}$. We are now done by the Artin-Tate lemma.

A related result was shown in [MS]: if $G$ is finite and $A$ is $k$-affine and Noetherian, then $A$ is finite over $A_{e}$, and $A_{e}$ is also affine and Noetherian.

\section{Enveloping Algebras of Lie COLOR Algebras}

In this last section, we apply some of our previous methods to the case of Lie color algebras; a basic reference on Lie color algebras is [BMPZ]. Throughout, $k$ will be a field and $G$ will be an abelian group with a fixed bicharacter $\langle\cdot \mid \cdot\rangle: G \times G \longrightarrow k^{*}$. Then a $G$-Lie color algebra is a $G$-graded $k$-space $L=\oplus_{g \in G} L_{g}$ with a bilinear map $[\cdot, \cdot]: L \otimes L \longrightarrow L$ satisfying

(i) $[x, y]=-\langle h \mid g\rangle[y, x]$,

(ii) $\langle g \mid l\rangle[x,[y, z]]+\langle l \mid h\rangle[z,[x, y]]+\langle h \mid g\rangle[y,[z, x]]=0$

for all homogeneous elements $x \in L_{g}, y \in L_{h}$, and $z \in L_{l}$. $U(L)$ will denote the universal enveloping algebra of $L$.

We first note that a graded version of Engel's theorem is known for Lie color algebras: if $L \subset \operatorname{End}(V)$, where $V$ is a finite-dimensional vector space, such that the homogeneous elements of $L$ act nilpotently on $V$, then $L$ is nilpotent on $V$. For, simply apply Jacobson's result to the set $S$ of homogeneous elements of $L$. This result was pointed out by Scheunert for the case of Lie superalgebras [S]: he says that "the usual proof works", and one assumes that he means to use weakly-closed sets. Recently Bergen and Grzeszczuk have extended Engel's theorem to the case when $V$ is not necessarily finite-dimensional. We give here a short proof of their result.

Theorem 3.1 ([BG]). Let $L$ be a finite-dimensional Lie color algebra and let $V$ be an $L$-module. If every homogeneous element of $L$ acts nilpotently on $V$, then $L$ acts nilpotently on $V$.

Proof. Since $L$ is finite-dimensional , $U(L)$ is Noetherian, and thus so is its image $\overline{U(L)}$ in $\operatorname{End}(V)$. If $S$ is the set of homogeneous elements in $L$, then $\bar{S}$ is weakly closed in $\overline{U(L)}$, and each $\bar{s} \in \bar{S}$ is nilpotent. Thus by Proposition 1.1, the associative algebra generated by $\bar{S}$ is nilpotent. Thus the image of $L$ in $\operatorname{End}(V)$ is certainly nilpotent.

We next prove a "generalized Nullstellensatz" for enveloping algebras of Lie color algebras, which extends known results for ordinary Lie algebras.

Theorem 3.2. Let $L$ be a finite-dimensional Lie color algebra and let $V$ be a $U(L)$ module of finite length. Then $\operatorname{End}_{U(L)}(V)$ is finite-dimensional over $k$. 
Proof. The proof follows the outline in [McR, Section 9.5] for ordinary Lie algebras. In fact the argument works for any Noetherian algebra $A$ such that

(i) $\quad A \otimes_{k} K$ is also Noetherian, for any field extension $K \supset k$;

(ii) $\operatorname{End}_{A}(V)$ is algebraic, for any simple $A$-module $V$.

For, $\operatorname{End}_{A}(V)=D$, a division ring, since $V$ is simple. If $C$ is a maximal subfield of $D$, then $C$ is a finitely-generated field extension of $k$ by [McR, Proposition 9.5.4]; this uses property (i). Then $C$ is finite-dimensional since $D$ is algebraic over $k$ by property (ii). Thus $D$ itself is finite-dimensional . To go from the case of $V$ simple to the case of $V$ having finite length, one may follow exactly the diagram in the proof of [McR, Theorem 9.5.5(ii)].

Thus it suffices to show (i) and (ii) for $U(L)$. Now (i) is true, since $U(L) \otimes K \cong$ $U(L \otimes K)$, which is still Noetherian. Also (ii) holds: this is known as Quillen's Lemma for ordinary Lie algebras, and the extension to Lie color algebras is shown in [BMPZ, p. 51].

\section{REFERENCES}

[BMPZ] Yu. A. Bahturin, A. A. Mikhalev, V. M. Petrogradsky, and M. V. Zaitsev, Infinite Dimensional Lie Superalgebras, De Gruyter, Berlin, 1992. MR 94b:17001

[BG] J. Bergen and P. Grzeszczuk, Engel type theorems for Lie color algebras, in Rings, Extensions, and Cohomology (A. Magid, ed), Marcel Dekker, New York, 1994.

[CR] M. Cohen and L. Rowen, Group-graded rings, Comm. in Algebra 11(1983), 1253-1270. MR 85b: 16002

[G] A. W. Goldie, Semiprime rings with maximum condition, Proc. London Math. Soc. 10(1960), 201-220. MR 22:2627

[H] B. Harris, Commutators in division rings, Proc. Amer. Math. Soc. 9(1958), 628-630. MR 20:3180

[J] N. Jacobson, Structure of Rings, Colloq. Publ. vol 37, Amer. Math. Soc., Providence, 1964. MR 36:5158

$[\mathrm{McR}] \quad$ J. C. McConnell and J. C. Robson, Non-commutative Noetherian Rings, WileyInterscience, New York, 1987. MR 89j:16023

[MS] S. Montgomery and L. W. Small, Some remarks on affine rings, Proc. Amer. Math. Soc. 98(1986), 537-544. MR 87k:16021

[NvO] C. Nastacescu and F. van Oystaeyen, Graded and Filtered Rings and Modules, LNM vol 758, Springer-Verlag, Berlin/New York, 1979. MR 80k:16002

[P] D. S. Passman, Infinite Crossed Products, Academic Press, San Diego, 1989. MR 90g:16002

[S] M. Scheunert, The Theory of Lie Superalgebras, LNM vol 716, Springer-Verlag, Berlin/New York, 1979. MR 80i:17005

Department of Mathematics, University of Southern California, Los Angeles, CalIFORNIA 90089

E-mail address: smontgom@math.usc.edu

Department of Mathematics, University of California, La Jolla, California 92093

E-mail address: 1wsmall@uscd.edu 